\title{
Modelling of monsoon rainfall for a mesoscale catchment in North-West India II: stochastic rainfall simulations
}

\author{
E. Zehe ${ }^{1}$, A. K. Singh ${ }^{2}$, and A. Bárdossy ${ }^{3}$ \\ ${ }^{1}$ Institute of Geoecology, University of Potsdam, Germany \\ ${ }^{2}$ Civil Engineering Department, Nirma University of Science \& Technology (NU) Ahmedabad - 382 481, India \\ ${ }^{3}$ Institute of Hydraulic Engineering, University of Stuttgart, Germany
}

Received: 15 July 2005 - Published in Hydrol. Earth Syst. Sci. Discuss.: 20 September 2005

Revised: 24 May 2006 - Accepted: 24 October 2006 - Published: 30 October 2006

\begin{abstract}
Within this study we present a robust method for generating precipitation time series for the Anas catchment in North Western India. The method employs a multivariate stochastic simulation model that is driven by a time series of objectively classified circulation patterns (CPs). In a companion study (Zehe et al., 2006) it was already shown that CPs classified from the 500 or 700 Hpa levels are suitable to explain space-time variability of precipitation in that area. The model is calibrated using observed rainfall time series for the period 1985-1992 for two different CP time series, one from the $500 \mathrm{Hpa}$ level and the over from the $700 \mathrm{Hpa}$ level, and 200 realizations of daily rainfall are simulated for the period 85-94. Simulations using the CPs from the $500 \mathrm{Hpa}$ level as input yield a good match of the observed averages and standard deviations of daily rainfall. They show furthermore good performance at the monthly scale. When used with the $700 \mathrm{Hpa}$ level CPs as inputs the model clearly underestimates the standard deviation and performs much worse at the monthly scale, especially in the validation period 93-94. The presented results give evidence that CPs from the $500 \mathrm{Hpa}$, level in combination with a multivariate stochastic model, make up a suitable tool for reducing the sparsity of precipitation data in developing regions with sparse hydrometeorological data sets.
\end{abstract}

\section{Introduction}

The PUB initiative, launched by Sivapalan et al. (2003), has identified a set of key targets for hydrological science. The most important thereof are the quantification and reduction of predictive model uncertainty. To our opinion, PUB shall not only be a catalyst to advance our hydrological understanding, but also can be a catalyst to reduce the number of poorly

Correspondence to: E. Zehe

(ezehe@rz.uni-potsdam.de) gauged basins. Of course building river gauges is expensive, laborious and therefore often not feasible. However, for the very important variable of rainfall we think it is possible to reduce the number of poorly gauged basins by employing downscaling methodologies. Especially in developing countries precipitation data sets are rare. If they are available, then they are often not in digital form, as in the case of our study of Monsoon rainfall in the Anas catchment, that is located in North-Western India. The very first step of this study was to collect data that were still on paper strips and turn them into digital data sets (1 month digitizing). This resulted in 10 years daily rainfall data for ten stations in the catchment. While this is already a major step forward, 10 years are not long enough for a thorough analysis of the precipitation in that area, because of the enormous seasonality and inter annual variability of the monsoon driven climate (Webster and Hoyos, 2004). The overall objective of the present study was therefore to suggest and test a methodology for extrapolating the available rainfall time series in a robust and reliable way, e.g. as basis for long term water resources and agricultural planning.

With robust we mean that the method is computationally efficient and based on data that are globally freely available. As re-analysis data of atmospheric and oceanic state variables and fluxes but also climate model runs are globally available on resolutions ranging from $2.5^{\circ}$ to $1^{\circ}$, downscaling methods (Wilby and Wilks, 1997) can help within this context (for a review of downscaling methods please refer to the companion paper Zehe et al., 2006). A major problem in the context of daily precipitation modelling is the spatial and temporal intermittency of precipitation i.e. the clustering of wet and dry days as well as the clustering of dry and wet areas in the target catchment. Approaches for stochastical modelling of daily precipitation range from multivariate regression models (Bürger, 2002; Huth, 1997; Huth and Kyselý, 2000), to Markov models for simulation precipitation occurrence and duration (Foufoula-Georgiou and

Published by Copernicus GmbH on behalf of the European Geosciences Union. 
Lettenmaier, 1987) and finally to stochastical models based on circulation patterns or weather types (Wilson et al., 1992; Katz and Parlange, 1996; Wilby and Wigley, 2000).

In the present study we adopt an empirical downscaling approach proposed by Stehlik and Bárdossy (2002) for stochastic rainfall simulations in the Anas catchment. The predictor variables are objectively classified circulation patterns from a suitable pressure level that have to be optimised for explaining the space-time variability in the target area. The time series of the circulation patterns serve in a second step as input variables to run a multivariate stochastic rainfall model. The link between daily rainfall and the $\mathrm{CP}$ is established by conditional rainfall probabilities and amounts respectively. In a companion study (Zehe et al., 2006) we already showed that pressure patterns are, despite the fact that the Coriolis parameter is half as large as compared to the middle latitudes, suitable and parsimoneous predictors for explaining precipitation variability in the Anas catchment. Within the present study we will shed light on the question of whether the two different CP classification schemes, one for the $500 \mathrm{HPa}$ level and the other for the $700 \mathrm{Hpa}$ level, yield suitable inputs to the multivariate stochastic rainfall model that shall reproduce the precipitation behaviour observed in the Anas catchment.

This paper is organized as follows. In section two we present the stochastic rainfall model, the statistical methods to analyse precipitation in the Anas catchment as well as the simulation strategy. Simulated and observed precipitation are compared in Sect. 3, with emphasis on daily and monthly scale rainfall properties, as well as the spatial structure and the autocorrelation structure. The study closes with discussion and conclusions in section 4. For details on the Anas catchment and the precipitation data base the reader might refer to the companion paper of Zehe et al. (2006).

\section{Methodology}

Stehlik and Bárdossy (2002) developed a methodology for generating spatio-temporal variable precipitation data using large scale daily pressure fields (simulated or observed) as well as local scale precipitation. The method consists of two main steps:

- An optimisation of fuzzy rules to classify pressure fields into circulation patterns (CPs), to explain the basin scale space-time variability of observed rainfall.

- A multivariate and stochastical generation of rainfall at different locations in the area of interest. The model is a conditional multivariate autoregressive rainfall model based on a transformed multivariate normal distribution. Rainfall is linked to the individual CP using conditional rainfall probability and amounts. The model accounts for the spatial covariance of daily precipitation is a function of the actual CP as well as of the day in the year. The annual cycles of the spatial covariance function and of the one day lag autocorrelation are described by means of a Fourier series.

The optimisation of two CP classification schemes for the $500 \mathrm{HPa}$ and the $700 \mathrm{HPa}$ level and their suitability for explaining the spatio-temporal variability of precipitation in the Anas catchment is described in the companion study (Zehe et al., 2006). Additional information on CP optimisation procedure may be found in Bárdossy et al. (2001). In the following we will briefly introduce the multivariate stochastical precipitation model.

\subsection{Stochastic rainfall model}

A major problem in the mathematical modelling of precipitation is spatial and temporal intermittency. Dry days occur with high probability, and on rainy days a continuous distribution describes the rainfall amounts at a selected location $u$. Therefore, a multi-site approach based on random variables with mixed distributions is required to describe the daily precipitation pattern. Within the time-space model suggested by Stehlik and Bárdossy (2002) the persistence of wet or dry days is a consequence of the persistence of the circulation patterns and the $\mathrm{CP}$ specific meteorological conditions, thus it is an input to the precipitation model.

Precipitation is linked to the individual $\mathrm{CP}$ through a conditional probability for the occurrence of a wet day $p(\mathrm{CP}(t))=\left(p\left(\mathrm{CP}(t), u_{1}\right), \ldots p\left(\mathrm{CP}(t), u_{M}\right)\right)$ and a conditional average amount $z(\mathrm{CP}(t))=\left(z\left(\mathrm{CP}(t), u_{1}\right), \ldots, z\left(\mathrm{CP}(t), u_{M}\right)\right) \quad$ which are derived from a set of precipitation time series observed at locations $u=\left(u_{1}, \ldots u_{M}\right)$ by means of frequency analysis. For a given $\mathrm{CP}$ the probability distribution, $Z(t, u)$ of daily rainfall amounts, $z=\left(z\left(t, u_{1}\right), \ldots z\left(t, u_{M}\right)\right)$ at a set of locations is of course multivariate skewed. Stehlik and Bárdossy (2002) relate $Z(t, u)$ to the positive branch of a multivariate normal process $N(t, u)$ by introducing a transformation within two steps. First they define a new variable $z^{\prime}=z-\boldsymbol{a}$, $z=\left(z_{u 1}, \ldots z_{u M}\right), \boldsymbol{a}$ is a constant vector, such that

$$
\begin{aligned}
& 1-\int_{-\infty}^{0} N(u, t) d z^{\prime}=1-\int_{-\infty}^{a} N(u, t) d z \\
& =p(\mathrm{CP}(t))=\left(p\left(\mathrm{CP}(t), u_{1}\right), \ldots, p\left(\mathrm{CP}(t), u_{M}\right)\right) \\
& d z=d z_{u 1} d z_{u 2} \ldots d z_{u M}
\end{aligned}
$$

This transformation assures that the integral of the positive branch of the shifted multivariate normal yields the observed conditional probability $p(\mathrm{CP}(t))$ for a wet day at each location $u_{1}, . ., u_{M}$. Second a suitable exponent $\beta$ has to be selected for each location $u_{1}, . ., u_{M}$ such that:

$$
\begin{aligned}
& \bar{z}\left(\mathrm{CP}(t), u_{i}\right) \\
& =\int_{0}^{\infty} z_{u i} Z\left(u_{i}, t\right) d z_{u_{i}}
\end{aligned}
$$




$$
\begin{aligned}
& =\int_{0}^{\infty} z_{u i}^{\prime}\left(N\left(u_{i}, t\right)\right)^{\beta} d z_{u_{i}}^{\prime} \\
& =\int_{a_{u i}}^{\infty} z_{u i}\left(N\left(u_{i}, t\right)\right)^{\beta} d z_{u_{i}}
\end{aligned}
$$

This assures that the positive branch of the power transformed multivariate normal process yields the conditional average daily rainfall amount $z(\mathrm{CP}(t))$ observed at the locations. By repeating this procedure for each $\mathrm{CP}$, the discrete discrete-continuous distribution of $Z(t, u)$ is related to the normal stochastic process $N(t, u)$.

$Z(t, u)=\left\{\begin{array}{cc}N^{\beta}(t, u) & \text { for } N(t, u)>0 \\ 0 & \text { for } N(t, u) \leq 0\end{array}\right\}$

Although in principle the conditional probability of a wet day $p(\mathrm{CP},(t), u)$ and the amount can be different for each day in the year, available rainfall time series are usually too short to evaluate $p(\mathrm{CP},(t), u)$ for each day in the year by frequency analysis. Stehlik and Bárdossy (2002) suggest the evaluation of $p(\mathrm{CP},(t), u)$ separately for each $\mathrm{CP}$ and location for each month in the year. After transformation of $Z$ into the multivariate normal process according to Eq. (1) and (2) the negative values of $N$ are declared as dry days at locations $u_{i}$, to reflect the intermittency of precipitation.

The stochastic rainfall model describes how the deviation from the CP conditional daily average normal process on a given Julian day of the year evolves from the corresponding deviation at the previous day:

$$
\begin{gathered}
N(t+1, u)-E\left(N(t+1 *, u) \mid \mathrm{CP}_{i}\right)=r(t *+1) \cdot(N(t, u) \\
\left.-E\left(N(t * u) \mid \mathrm{CP}_{i}\right)\right)+\mathbf{C}_{\mathrm{CP} i}(t *+1) \cdot \boldsymbol{\Psi}(t *, u)
\end{gathered}
$$

where $E$ denotes the expectation value conditioned to the $\mathrm{Ju}$ lian day and the $\mathrm{CP}_{i}, r$ is the 1 day lag autocorrelation function that is assumed to be constant in space but is a function of the Julian day in the year (annual cycle). The matrix $\mathbf{C}$ is related to the spatial covariance matrix and the one day lag spatial covariance matrix of the multivariate normal process as described in Stehlik and Bárdossy (2002) or in Brás and Rodrigue-Iturbe (1985). It depends on the circulation pattern and on the annual cycle. The vector $\boldsymbol{\Psi}$ denotes a random vector of independent normally distributed random numbers with zero mean and standard deviation 1. With Eq. (4) and the link between the multivariate normal process and multivariate rainfall distribution $Z$ in Eq. (3) the rainfall model is totally described.

The autocorrelation function in $r$ is defined independent of the rainfall stations and is modelled by means of a Fourier analysis. In the study of Stehlik and Bárdossy (2002) that was conducted for two catchments in Germany in and Greece, the first three harmonics were found to be sufficient for explaining the annual cycle.

For computing the matric $\mathbf{C}$ in Eq. (6) as well as for characterising the multivariate normal process it is necessary to estimate the spatial covariance function, cov, from the rain gauge data. Cov is a function of the month of the year and the $\mathrm{CP}$ and we use an exponential spatial covariance function:

$\operatorname{cov}(Z(x), Z(y))=C_{0} \exp \left(\frac{-d(x, y)}{\lambda}\right)$

where $d$ is the distance and $C_{0}$ and the correlation length $\lambda$ is a functions of month in the year and the CP.

\subsection{Statistical measures for model performance and model calibration}

\subsubsection{Statistical measures for model performance}

In order to check the models capability to reproduce the observed monsoon precipitation in the Anas catchment we selected at the daily scale the average and standard deviation of the daily rainfall amounts. At the monthly scale we compared the simulated and observed annual cycles as well as the time series of simulated and observed rainfall. Furthermore, we compared the long term average spatial covariance structure by estimating the variogram as follows. In the first step we calculated the cross correlation for each pair of the ten rain gauges for the simulation and the observations, respectively. By plotting the cross correlation values against the distance between the pairs we obtain a spatial correlogram. Assuming stationarity of the increments one minus the cross correlation values plotted against the distances gives an estimate of the variogram, which sheds light on the average spatial structure of the monsoon precipitation in this area. The lag step for calculation the empirical variogram was set to $12000 \mathrm{~m}$. As theoretical estimator we selected an exponential variogram function. Finally, to shed light on the memory of the monsoon rainfall process we calculated the autocorrelation of the simulated and observed rainfall time series.

\subsection{Model calibration and simulation variants}

To estimate the model parameters especially the matrix $\mathbf{C}$, that depends on the month and the $\mathrm{CP}$, one needs a data set of 10 years or more especially when the inter-annual variability of precipitation is as high as in the Anas catchment. Within the 10 year observation period the annual precipitation sums range from 350 to $1300 \mathrm{~mm}$ ! On the other hand, it is necessary to test the model using an independent period; hence we selected an 8 year calibration period 85-92 and 2 year validation period 93-94. The model was calibrated using the $\mathrm{CP}$ time series from both classification regimes for the $500 \mathrm{Hpa}$ and the 700 Hpa level (Zehe et al., 2006). Following Stehlik and Bárdossy (2002) we used the first 3 harmonics for describing the annual cycles of the autocorrelation function. However, the calibration and estimation of Fourier coefficients was only carried out for the period May-October. This is because rainfall data in the Anas catchment were only collected in this period. Hence, we assumed precipitation out- 
Table 1. Observed and simulated daily average daily rainfall amount in mm (upper three rows) as well as observed and simulated standard deviation (stdev., in $\mathrm{mm}$ ) of daily rainfall totals (lower three rows) for the ten rain gauges.

\begin{tabular}{lcccccccccc}
\hline & 1 & 2 & 3 & 4 & 5 & 6 & 7 & 8 & 9 & 10 \\
\hline Mean obs. & 5.14 & 5.09 & 5.34 & 5.57 & 6.10 & 4.76 & 5.75 & 5.20 & 5.02 & 6.64 \\
Mean 700 Hpa & 6.46 & 5.49 & 5.51 & 6.25 & 6.26 & 5.21 & 7.27 & 5.66 & 5.31 & 7.23 \\
Mean 500 Hpa & 5.50 & 5.34 & 5.15 & 6.21 & 6.09 & 5.06 & 5.56 & 5.90 & 5.39 & 7.00 \\
Stdev. Obs. & 15.9 & 16.3 & 14.7 & 16.7 & 19.6 & 14.9 & 17.6 & 14.7 & 14.8 & 18.9 \\
Stdev. 700 Hpa & 14.8 & 15.2 & 12.4 & 13.9 & 16.3 & 12.9 & 16.2 & 13.9 & 12.5 & 15.7 \\
Stdev. 500 Hpa & 15.6 & 15.8 & 14.4 & 16.5 & 19.0 & 15.2 & 16.1 & 15.1 & 14.3 & 18.3
\end{tabular}

1 = Jhabua, 2 = Ranapur, $3=$ Udaigarh, $4=$ Amba, $5=$ Rama, $6=$ Meghnagar, $7=$ Thandla, $8=$ Bhabra, $9=$ Sardapur, $10=$ Petlabad
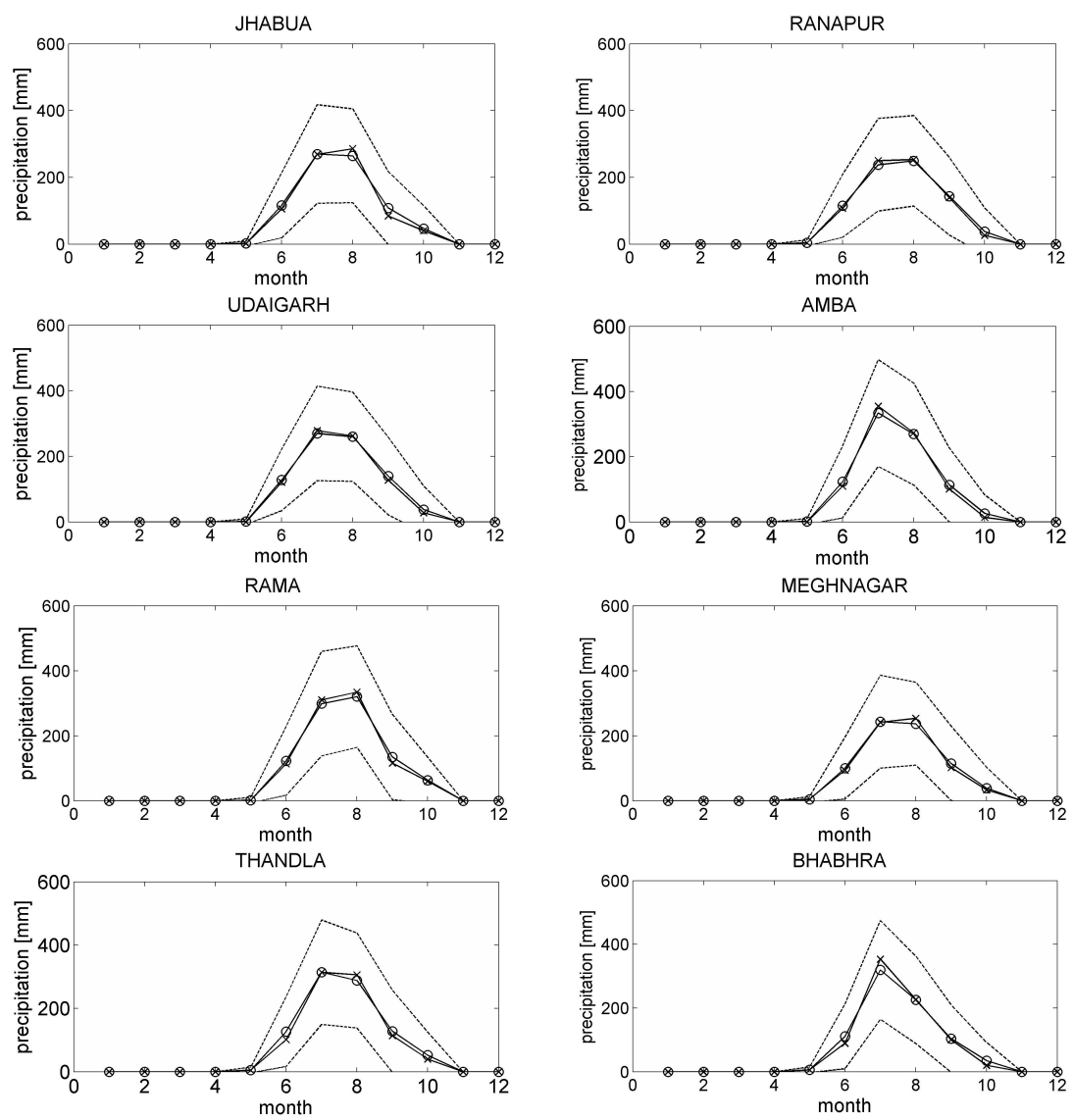

Fig. 1. Annual cycles of monthly precipitation sums for 8 rain gauges for the simulation driven by the CPs from the $500 \mathrm{Hpa}$ pressure level. The solid line with the crosses is the observation the solid line the circles denote the average from the 200 realisations. The dashed lines are average plus/minus the standard deviation computed based on the 200 realisations.

side this period to be zero. Rainfall probabilities and Fourier coefficients outside were therefore set to zero! In general we performed a total number of 200 simulations for both classification schemes. Due to the short validation period all the statistical properties employed for the model test were computed for the whole observation period 1985-1994.

\section{Results}

Table 1 lists the averages and standard deviations of daily rainfall amounts observed at the 10 stations compared to the corresponding values derived from the simulations. In general the values from the simulations for the $500 \mathrm{Hpa}$ level CP show a much better match with the observations. Deviations 

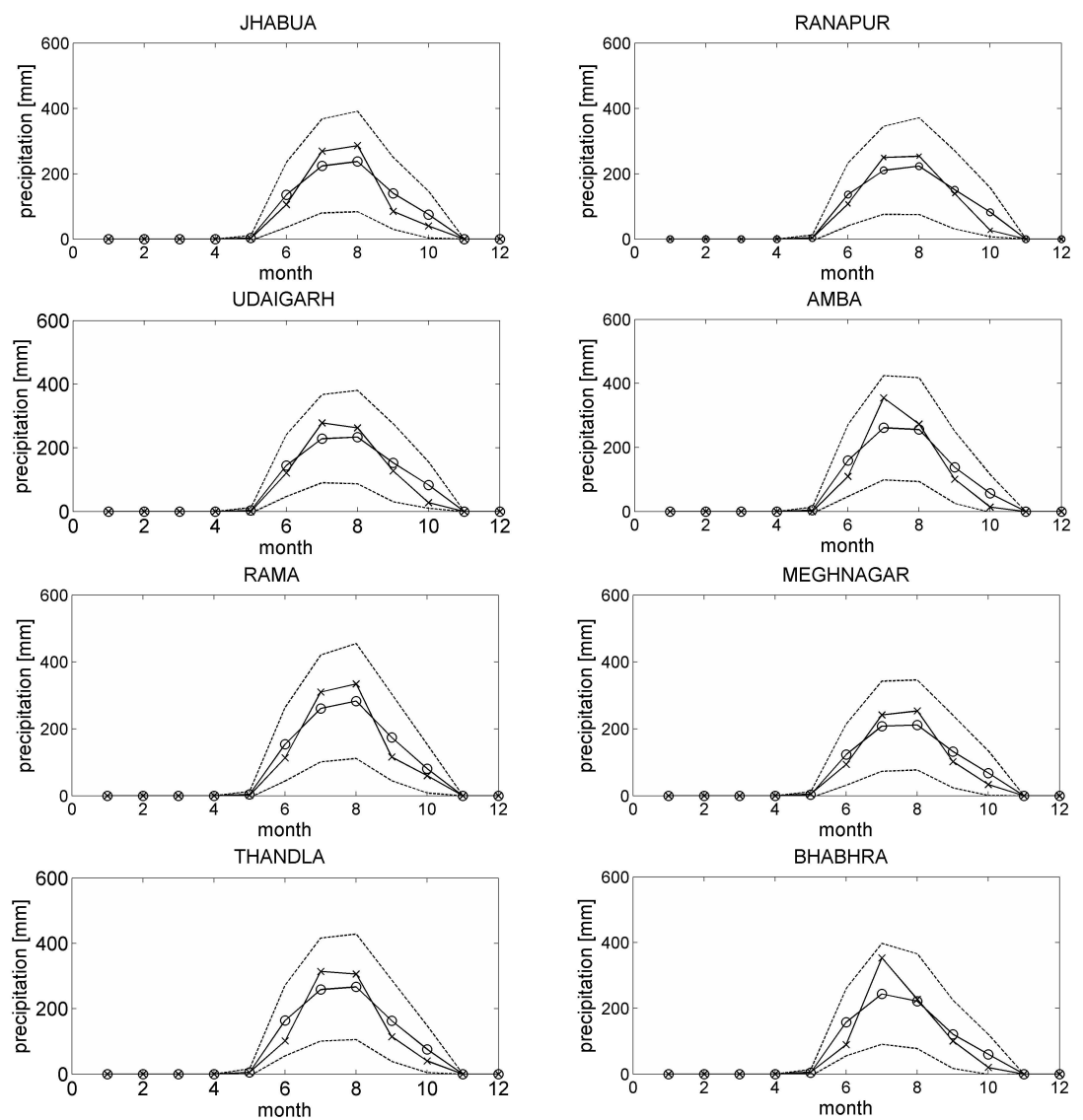

Fig. 2. Annual cycles of monthly precipitation sums for 8 rain gauges for the simulation driven by the CPs from the $700 \mathrm{Hpa}$ pressure level. The solid line with the crosses is the observation the solid line the circles denote the average from the 200 realisations. The dashed lines are average plus/minus the standard deviation computed based on the 200 realisations.

from the observed averages and standards deviation are entirely within the $95 \%$ confidence limit. Consistently with the findings of the companion paper (Zehe et al., 2006), the simulations driven by the CPs from the $700 \mathrm{Hpa}$ level exhibit a systematic underestimation of the variability of daily rainfall. It leads furthermore to a clear systematic overestimation of daily average rainfall rates with a maximum value of $1.7 \mathrm{~mm}$ at the station Thandla.

Moving on to the monthly scale the simulations using the 500 Hpa classification scheme yields a good match with the observed annual cycle at all stations, as shown for 8 stations in Fig. 1. When compared to the annual cycle simulated using the $700 \mathrm{Hpa} \mathrm{CPs}$ one can see, that in this case the model overestimated the average monthly rainfall sums in the period from June to August and slightly underestimates precipitation in the beginning and the end of the monsoon period. However, all the deviations fall within range of the standard deviation derived from the 200 simulations.

As can be seen from Fig. 3 the model yields a good match of the observed time series of monthly rainfall total in the calibration period 85-92, when driven with the CPs from the $500 \mathrm{Hpa}$ pressure level. During the validation period 93-95 the model clearly under-predicts monthly rainfall in June 1993 and August 1995, however, the deviations are still within the $95 \%$ confidence limit. When the CPs from the $700 \mathrm{Hpa}$ level are used as inputs the model performs much worse, especially at stations Bhabra, Amba and Udaighar (Fig. 4). The observed precipitation values fall outside the 95\% confidence limit several times inside the calibration period as well as in June 1993 and August 1995.

Consistently, when driven with the CP time series from the $500 \mathrm{Hpa}$ level the model yields a much better estimate of the average spatial correlation structure of the monsoon precipitation. As can be seen in Fig. 5 the experimental variograms are very similar and the fit of an exponential variogram function yields for observation and simulation identical values for nugget $(0)$, sill $(0.6)$ and range $(25000 \mathrm{~m})$. Please note that the values for nugget and sill are normalised with the station variance. When driven with CPs from the $700 \mathrm{Hpa}$ level spatial pattern of simulated rainfall shows a worse match: the nugget $(0.1)$ is overestimated and the sill $(0.5)$ is underestimated. 

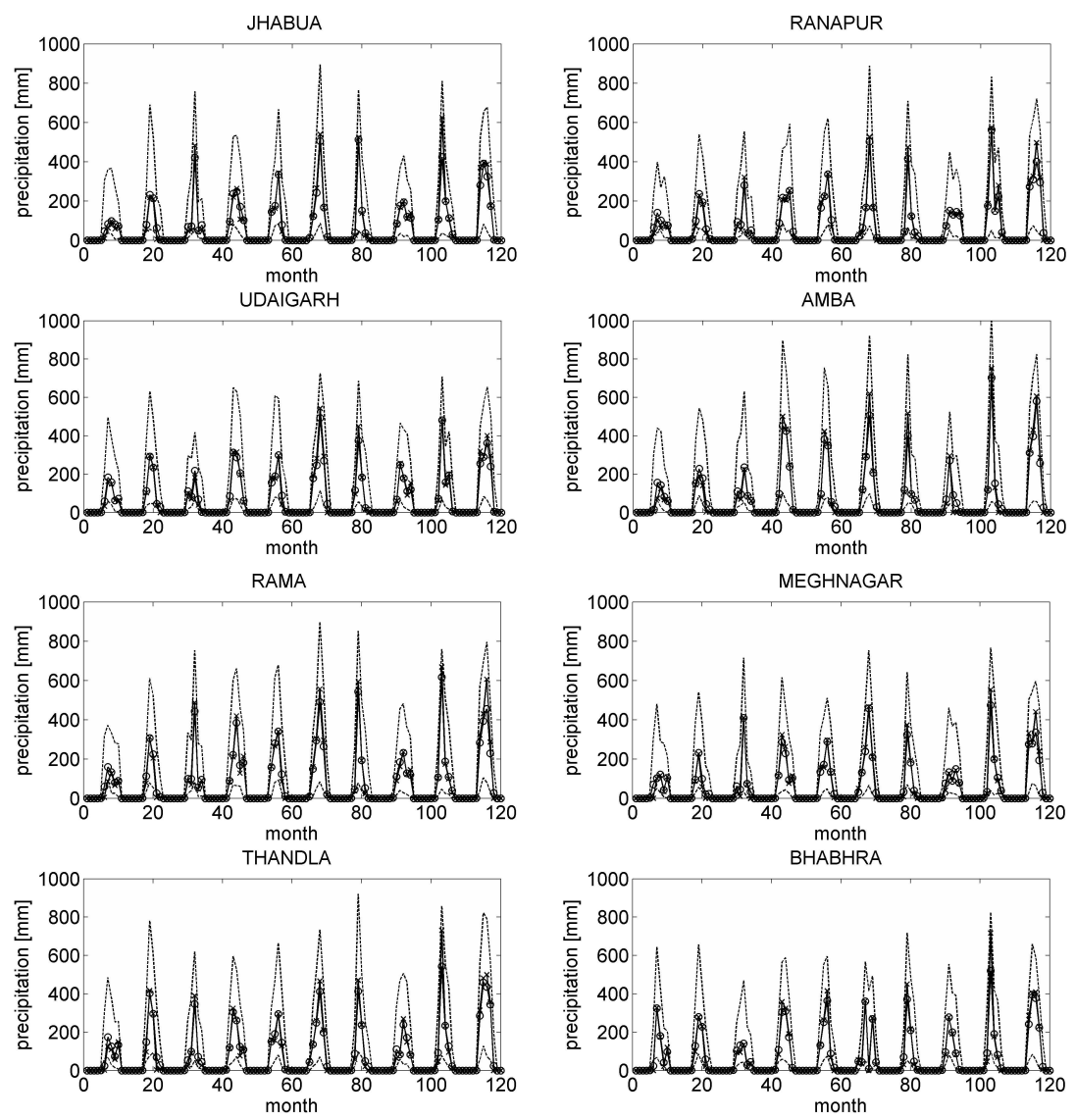

Fig. 3. Time series of observed and simulated monthly totals for the period 1985-1994 for the classification scheme at the 500 Hpa level. The solid line with the crosses is the observation the solid line the circles denote the average from the 200 realisations. The dashed lines are the $95 \%$ confidence intervals derived from the 200 realisations.

Figure 6 compares finally the autocorrelation function from both simulations to the long term average autocorrelation computed for the 10 stations (crosses). In general there is no difference between the autocorrelation functions for both classification schemes. Due to the assumption of an exponential autocorrelation, values are slightly overestimated for lags between one to four days. In general the matching is good. It can be seen furthermore that the spatial variability between the autocorrelation functions is quite small. Interestingly the autocorrelation at all stations shows a local maximum at a lag of 4-6 days.

\section{Discussion and conclusions}

The results presented in this and the companion study (Zehe et al., 2006) give evidence that objectively classified circulation patterns are a) suitable for explaining space-time variability of precipitation in the Anas catchment in North Western India and b) allow reasonable stochastic rainfall simulations which match the observed precipitation data well, espe- cially at the monthly time scale. It is interesting to note that the model when driven with CPs from the $700 \mathrm{Hpa}$ level exhibits a systematic underestimation of the daily rainfall variability when compared to the case when the model is driven with CPs from the $500 \mathrm{Hpa}$. The results from the companion paper (Zehe et al., 2006) already indicated that the conditional rainfall probabilities of the $700 \mathrm{Hpa}$ classification scheme allow a worse separation between wet and dry larges scale meteorological conditions than the corresponding values from the classification scheme for the $500 \mathrm{Hpa}$ level. Consistently at the monthly time the model yields clearly better results when used with CPs from the $500 \mathrm{Hpa}$ pressure level. Due to the weaker Coriolis force one might expect that pressure patterns located closer to the ground have a stronger influence on tropical circulation than pressure at the $500 \mathrm{Hpa}$ level. However, the Anas catchment is located at $24^{\circ} \mathrm{N}$ and the Coriolis parameter is already half as large as compared to the mid latitudes. This seems to be large enough to influence circulation in this region.

One important goal of this study was to propose a robust method for reducing data shortage in developing areas 

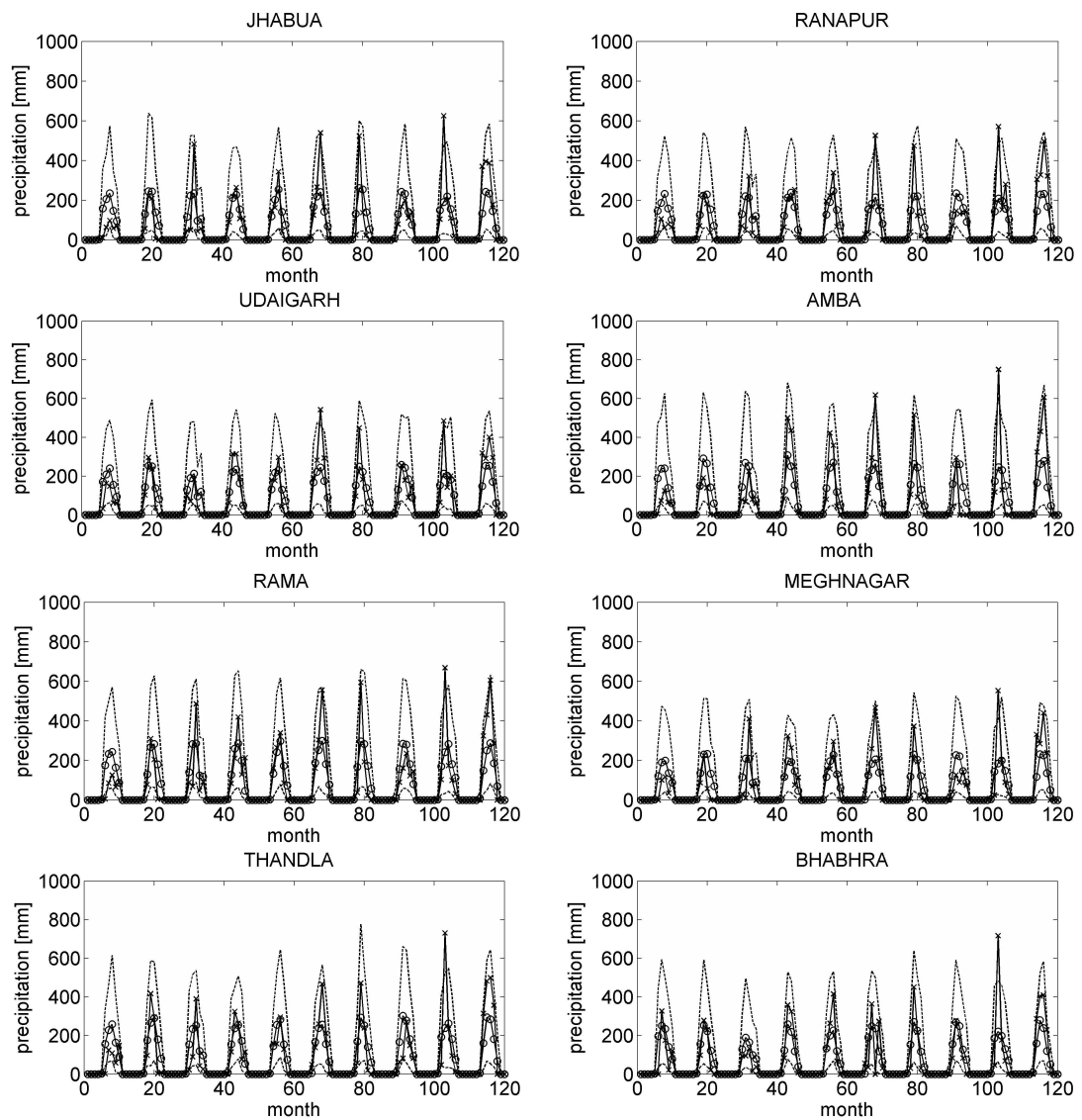

Fig. 4. Time series of observed and simulated monthly totals for the period 1985-1994 for the classification scheme at the 700 Hpa level. The solid line with the crosses is the observation the solid line the circles denote the average from the 200 realisations. The dashed lines are the $95 \%$ confidence intervals derived from the 200 realisations.

with sparse hydrological datasets. The presented approach yields a good estimate for precipitation at the monthly scale, which is the first step towards water resources planning. The method is robust as NCEP pressure data are globally available for CP optimisation and classification and since it is computationally efficient. The stochastic model runs on a PC, computation time for 100 realisations of 10 years length is 30 min. As shown in Zehe et al. (2006) the CP time series is a scalar predictor that embeds the information on the spatial pressure pattern i.e. important locations of depressions and heights with it. Thus, the proposed method is indeed robust and suitable for generating precipitation time series for the past periods where no observations are available. As the model accounts for the spatial covariance structure of the precipitation it may also be employed to interpolate precipitation data to locations inside the catchment e.g. by using external drift Kriging (Stehlik and Bárdossy, 2002). Hence, we may conclude that the model is suitable for the PUB issue.

The proposed method may in principle be extended to account for additional predictors for Monsoon rainfall such as SST (sea surface temperature) as suggested by Harzallah and Sadourny, 1999) or Clark et al. (1999) for example by introducing rainfall probabilities and amounts conditional upon the CP and SST anomalies. However, in case of projections into the future one has to assume, as for all empirical approaches, that the predictor-predictant relation is invariant in the presence of climate change. This is a very strong assumption should be carefully investigated e.g. by combining dynamical and empirical downscaling approaches.

Edited by: M. Sivapalan

\section{References}

Bárdossy, A., Stehlik, J., and Caspary, H.-J.: Generating of areal precipitation series in the upper Neckar catchment, Phys. Chem. Earth (B), 26, 9, 683-687, 2001.

Bürger, G.: Selected precipitation scenarios across Europe. J. Hydrol., 262, 99-110, 2002. 


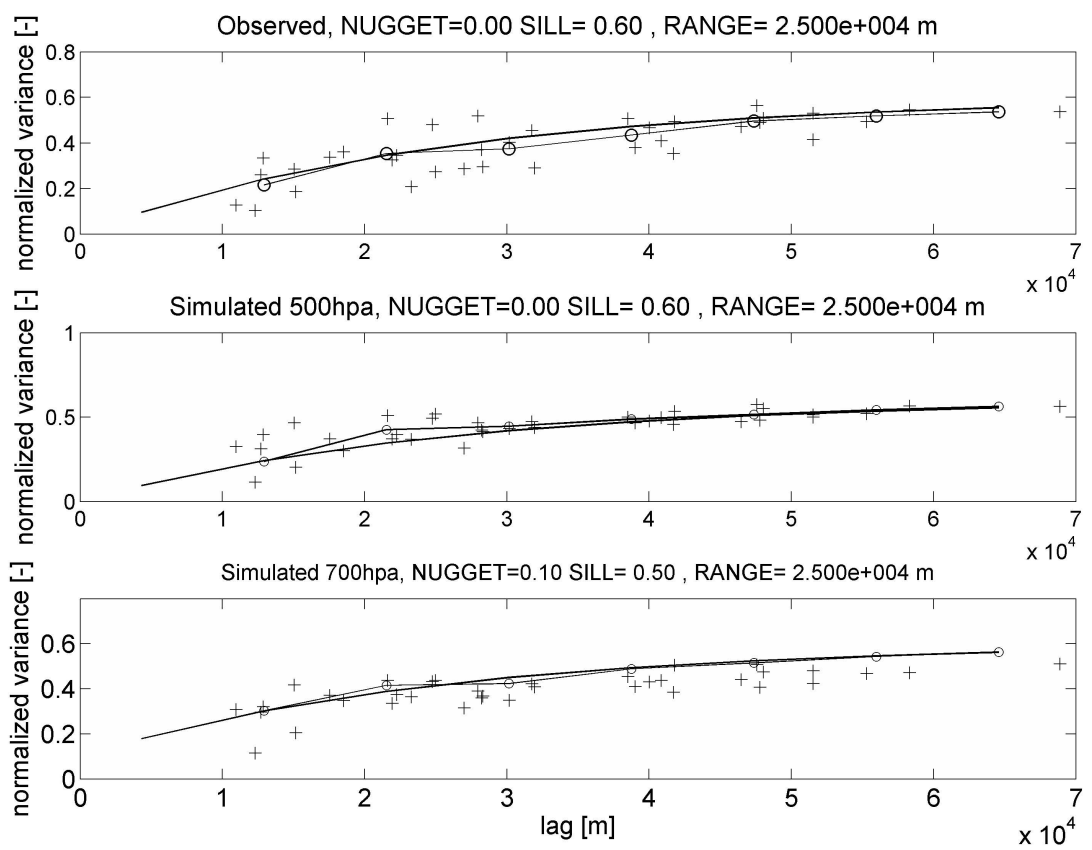

Fig. 5. Average variogram structures of the observed daily rainfall (upper panel), the simulation driven with the $500 \mathrm{Hpa}$ level CPs (middle panel) and the simulation driven with the $700 \mathrm{Hpa}$ level CPs (lower panel). The crosses mark the experimental variogram cloud i.e. 1- the cross correlation value between two stations, plotted against the distance separating the stations. The solid line with the circles marks the experimental variogram computed by averaging the cloud values in the lag classes of $12000 \mathrm{~m}$ width. The solid line is exponential variogram fitted to the experimental variogram.

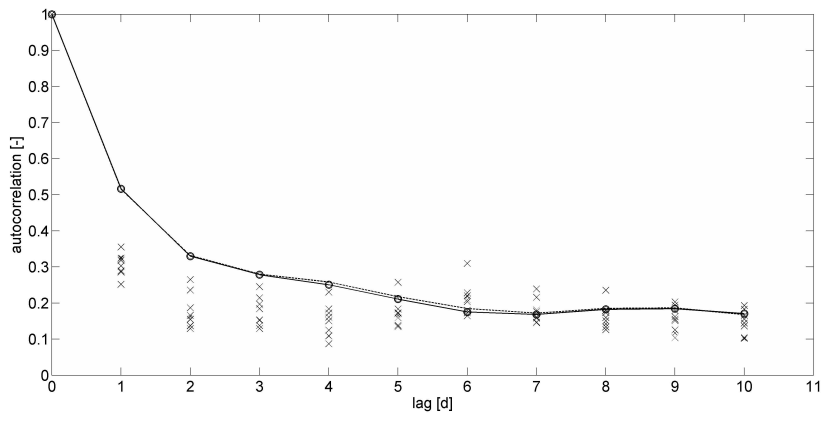

Fig. 6. Average daily autocorrelation of the simulated daily precipitation for the $500 \mathrm{Hpa}$ level (solid line with circles) and the $700 \mathrm{Hpa}$ level (dashed). The crosses mark the autocorrelation values observed at the 10 stations for time lags ranging from 1-10. Please note that the model per definition assumes a spatial uniform autocorrelation.

Clark, C. O, Cole, J. E., and Webster, P. J.: Indian ocean SST and Indian summer rainfall: Predictive relationships and their decadal variability, J. Clim., 13, 2503-2519, 1999.

Foufoula-Georgiou, E. and Lettenmaier, D.: A markov renewal model for rainfall occurrences, Water Resour. Res., 23, 875-884, 1987.

Harzallah, A. and Sadourny, R.: Observed lead lag relationships between Indian summer monsoon and some meteorological variables, Clim. Dyn., 13, 635-648, 1999.

Hulme, M.: An intercomparision of model and observed global precipitation climatologies, Geophys. Res. Lett., 18, 1715-1718, 1991.

Huth, R.: Potential of continental scale circulation for the determination of local scale surface variables, Theor. Appl. Climatol. 56, 165-186, 1997.

Huth, R. and Kyselý, J.: Constructing site specific climate change scenarios on a monthly scale using statistical downscaling, Theor. Appl. Climatology, 66, 13-27, 2000.

Katz, R. W. and Parlange, M. B.: Effects of an index of atmospheric circulation on stochastic properties of precipitation, Water Resour. Res., 29, 2335-2344, 1993.

Sivapalan, M., Takeuchi, K., Franks, S. W., Gupta, V. K., Karambiri, H., Lakshmi, V., Liang, X., McDonnell, J. J., Mendiondo, E. M., O’Connel, P. E., Oki, T., Pomeroy, J. W., Schertzer, D., Uhlenbrook, S., and Zehe, E.: IAHS decade on Predictions of Ungaged Basins (PUB): Shaping an exciting future for the hydrological sciences, Hydrol. Sci. J., 48(6), 857-879, 2003.

Stehlik, J. and Bárdossy, A.: Multivariate stochastic downscaling model for generating daily Rainfall series based on atmospheric circulation, J. Hydrol., 256, 120-141, 2002.

Webster, P. J. and Hoyos, C.: Prediction of monsoon rainfall and river discharge on 15-30 day time scales, Bull. Amer. Meteor. Soc., 85, 1745-1767, 2004.

Wilby, R. L. and Wigley, T. M. L.: Precipitation predictors for downscaling - observed and general circulation model relationships, Int. J. Climatol., 20, 641-661, 2000.

Wilby, R. L. and Wigley, T. M. L.: Downscaling general circulation model output: a review of methods and limitations, Prog. Phys. Geogr., 21, 530-548, 1997. 
Wilson, L. L., Lettenmaier, D. P., and Skyllingstaed, E.: A multiple stochastic daily precipitation model conditional on large scale circulation patterns, J. Geophys. Res., 97, 2791-2801, 1992.
Zehe, E., Singh A. K., and Bárdossy, A.: Modelling of monsoon rainfall for a mesoscale catchment in North-West India I: assessment of objective circulation patterns, Hydrol. Earth Syst. Sci., 10, 797-806, 2006,

http://www.hydrol-earth-syst-sci.net/10/797/2006/. 